Gastroenterologe 2011 · 6:369-370

DOI 10.1007/s11377-011-0539-8

○) Springer-Verlag 2011

\author{
G. Gerken ${ }^{1} \cdot$ R. Jakobs ${ }^{2}$ \\ ${ }^{1}$ Klinik für Gastroenterologie und Hepatologie, Universitätsklinikum Essen \\ ${ }^{2}$ Medizinische Klinik C, Klinikum der Stadt Ludwigshafen
}

\title{
Perspektiven der Palliativmedizin in der Gastroenterologie
}

Die Betreuung alter und hochbetagter Menschen nimmt generell weltweit und insbesondere in den hochentwickelten Industrienationen kontinuierlich zu. Hierzu tragen sicher die höhere Lebenserwartung und die verbesserte Medizin in der modernen Gesellschaft entscheidend bei.

Das Bewusstsein für die Problematik der palliativmedizinischen Versorgung in Deutschland wurde gerade aktuell innerhalb der Ärzteschaft im Rahmen der Entschließungen des 114. Deutschen Ärztetages in Kiel geschärft. Zudem wurde die Palliativmedizin im Jahre 2009 als Pflicht-, Lehr- und Prüfungsfach in die Approbationsordnung für Ärzte aufgenommen.

Auch die Gastroenterologie wird sich diesen Herausforderungen stellen müssen. In Zukunft ist es sicher notwendig, palliativmedizinische Konzepte im Rahmen der gesundheitlichen Versorgung genau zu definieren und zu etablieren. Häufig wird Palliativmedizin immer noch mit Schmerz- und Sterbemedizin in ausweglosen Situationen gleichgesetzt. Auch ist Palliativmedizin weder beschränkt auf onkologische Patienten noch Ersatz für Rationalisierungs- oder Rationierungsüberlegungen.

Zur Palliativmedizin gehört somit nicht nur die Linderung körperlicher Symptome, sondern auch eine ganzheitliche Herangehensweise zur Verbesserung der Lebensqualität von Patienten in ihrer letzten Lebensphase. In Deutschland ist davon auszugehen, dass ca. $10-15 \%$ aller Menschen im Finalstadium ihres Lebens eine spezialisierte Palliativversorgung benötigen.
Gerade die frühzeitige Einbindung der Palliativmedizin bei fortgeschrittenen Tumorerkrankungen im Rahmen des Konzeptes der Therapiezieländerung führt oft $\mathrm{zu}$ einer verbesserten Lebensqualität der Patienten, zur Kosteneinsparung und sogar zu einer signifikanten Lebensverlängerung. Dies alles ist jedoch nur erreichbar durch multiprofessionelle Interventionen der Palliativmedizin zur Symptomenkontrolle nicht nur durch neue Konzepte der Schmerzmitteltherapie, sondern auch durch minimalinvasive interventionelle Palliativkonzepte. Daneben spielt natürlich die psychosoziale Versorgung weiterhin eine große Rolle.

\section{(2) Bei ca. $80 \%$ der palliativ- medizinischen Patienten treten gastrointestinale Symptome auf}

Das von den Herausgebern in Zusammenarbeit mit den Autoren erarbeitete hier vorliegende Heft gibt eine aktuelle Bestandsaufnahme verschiedener Probleme der palliativmedizinischen Versorgung aus gastroenterologischer Sicht. Insbesondere widmen sich diese Beiträge den spezifischen Inhalten der Palliativmedizin unter dem besonderen Aspekt der Gastroenterologie. So treten bei ca. $80 \%$ aller palliativmedizinischen Patienten gastrointestinale Symptome auf, unabhängig von der Primärdiagnose. Damit ist die Symptomenkontrolle vorrangiges Ziel der Palliativmedizin.

Auch die Ernährung hat in der Palliativmedizin eine übergeordnete Bedeu- 
tung, gerade im Rahmen des Therapiezielwechsels. Sie sollte in abgestuften Vorgehensweisen kompetent eingesetzt werden, von der oralen Ernährung über die enterale Sondennahrung bis zur häuslichen parenteralen Ernährung und zur Beherrschung spezifischer Essensprobleme in der terminalen Krankheitsphase.

Für die Aufrechterhaltung der Lebensqualität und die Achtung der Menschenwürde am Lebensende sowie bei unheilbaren Erkrankungen spielt die Beherrschung der Schmerztherapie - sowohl systemisch als auch interventionell - eine herausragende Rolle. Besonders die Kombination mit endoskopischen Verfahren, z. B. bei der Beherrschung maligner Obstruktionen im Gastrointestinaltrakt, erfordert gastroenterologische Fachkompetenz. Mit interventionellen technischen Überbrückungsverfahren lässt sich die Effizienz der Palliativmedizin steigern, um Schmerzmittelgebrauch zu reduzieren, Lebensqualität zu verbessern, Krankenhausaufenthalte zu verkürzen und Kosten zu verringern.

Gerade die Diskussionen über den Umgang mit Sterben und Tod bedeuten große Herausforderungen für jeden Arzt und natürlich auch für jeden interventionell arbeitenden Gastroenterologen. Hierbei ergeben sich ethische Spannungsfelder, die es erforderlich machen, sich aktiv auch mit rechtlichen ethischen und moralischen Problemen aus ärztlicher Sicht auseinanderzusetzen. Somit müssen neben fachmedizinischen Kenntnissen, die in der Palliativmedizin Anwendung finden, auch hohe kommunikative Kompetenz, Empathie und das Wissen um die juristischen und ethischen Pflichten wie auch die Grenzen der Behandlung unabdingbar in unsere tägliche ärztliche Praxis einfließen.

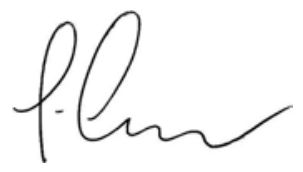

G. Gerken

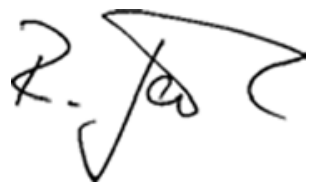

R. Jakobs

\section{Korrespondenzadressen}

Prof. Dr. G. Gerken

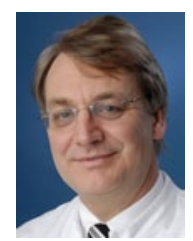

Klinik für Gastroenterologie und Hepatologie, Universitätsklinikum Essen Hufelandstrase 55, 45122 Essen

Guido.Gerken@uk-essen.de

Prof. Dr. R. Jakobs

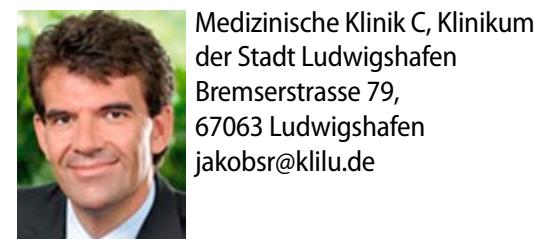

Eva-Luise-Köhler-Forschungspreis für Seltene Erkrankungen 2012

Die Eva Luise und Horst Köhler Stiftung für Menschen mit Seltenen Erkrankungen vergibt in enger Kooperation mit der Allianz Chronischer Seltener Erkrankungen (ACHSE e.V.) zum fünften Mal einen Preis für Forschungsprojekte, die sich seltenen Erkrankungen widmen. Der mit 50.000 Euro dotierte Forschungspreis ist nach der Schirmherrin der ACHSE, Frau Eva Luise Köhler, benannt.

Allein in Deutschland sind bis zu 4 Millionen Menschen von einer der über 5.000 Seltenen Erkrankungen betroffen. Viele bedeuten für den Patienten eine deutliche Verminderung der Lebensqualität und der Lebensdauer. Durch den Mangel an Forschung fehlen Medikamente und Therapien. Mit dem Eva-Luise-Köhler-Forschungspreis soll die Durchführung bzw. Anschubfinanzierung eines am Patientennutzen orientierten Forschungsprojektes im Bereich der Seltenen Erkrankungen ermöglicht werden. Der Forschungspreis macht zudem Wissenschaft, Industrie und Gesellschaft darauf aufmerksam, dass die Forschung zu den vordringlichsten Aufgaben der Gesundheitspolitik gehören sollte. Bewerbungsschluss für den Forschungspreis ist der 3. Oktober 2011. Am 28.

Februar 2012 erfolgt in Berlin die feierliche Vergabe an den oder die Preisträger. Interessierte Wissenschaftler erhalten detaillierte Informationen zur Bewerbung und die Bewerbungsunterlagen unter www. achse-online.de.

Quelle: Allianz Chronischer Seltener Erkrankungen (ACHSE e. V.), www.achse-online.de 